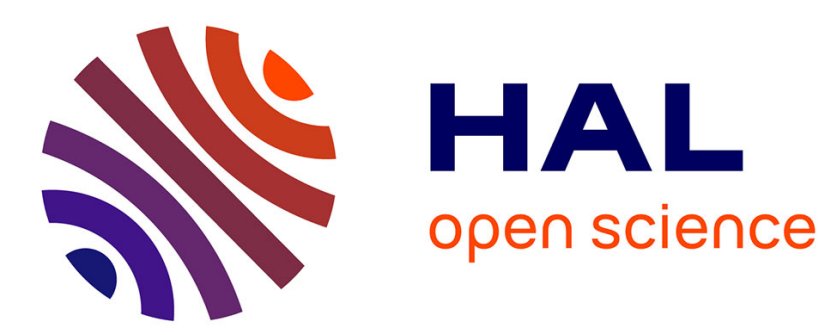

\title{
Optimal switching from competition to cooperation: a preliminary exploration
}

Raouf Boucekkine, Carmen Camacho, Benteng Zou

\section{To cite this version:}

Raouf Boucekkine, Carmen Camacho, Benteng Zou. Optimal switching from competition to cooperation: a preliminary exploration. 2020. halshs-02434786

\section{HAL Id: halshs-02434786 \\ https://shs.hal.science/halshs-02434786}

Preprint submitted on 10 Jan 2020

HAL is a multi-disciplinary open access archive for the deposit and dissemination of scientific research documents, whether they are published or not. The documents may come from teaching and research institutions in France or abroad, or from public or private research centers.
L'archive ouverte pluridisciplinaire HAL, est destinée au dépôt et à la diffusion de documents scientifiques de niveau recherche, publiés ou non, émanant des établissements d'enseignement et de recherche français ou étrangers, des laboratoires publics ou privés. 


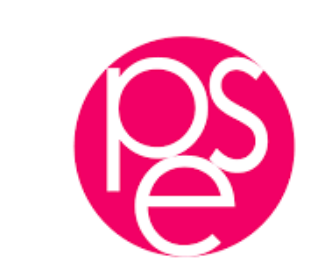

PARISSCHOOLOFECONOMICS
ECOLED'ECONOMIEDEPARIS

WORKING PAPER № $2020-01$

Optimal switching from competition to cooperation: a preliminary exploration

\author{
Raouf Boucekkine \\ Carmen Camacho \\ Benteng Zou
}

JEL Codes:

Keywords: Cooperation, competition, dynamic games, multi-stage optimal control

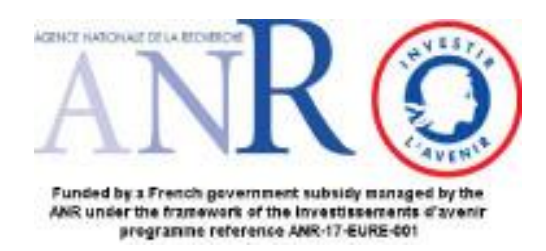




\title{
Optimal switching from competition to cooperation: a preliminary exploration
}

\author{
Raouf Boucekkine* $\quad$ Carmen Camacho $^{\dagger} \quad$ Benteng Zou $^{\ddagger}$
}

December 25, 2019

\begin{abstract}
In this paper, we tackle a generic optimal regime switching problem where the decision making process is not the same from a regime to another. Precisely, we consider a simple model of optimal switching from competition to cooperation. To this end, we solve a twostage optimal control problem. In the first stage, two players engage in a dynamic game with a common state variable and one control for each player. We solve for open-loop strategies with a linear state equation and linear-quadratic payoffs. More importantly, the players may also consider the possibility to switch at finite time to a cooperative regime with the associated joint optimization of the sum of the individual payoffs. Using theoretical analysis and numerical exercises, we study the optimal switching strategy from competition to cooperation. We also discuss the reverse switching.
\end{abstract}

Keywords: Cooperation, competition, dynamic games, multi-stage optimal control. JEL classification:

*Aix-Marseille University (Aix-Marseille School of Economics and Institute for Advanced Study), CNRS, and EHESS. 2 rue de la charité, 13002 Marseille, France. E-mail: Raouf.Boucekkine@univ-amu.fr

${ }^{\dagger}$ Paris Sciences et Lettres, Paris School of Economics and CNRS, France. E-mail: carmen.camacho@psemail.eu.

$\ddagger$ University of Luxembourg. 6, rue Richard Coudenhove-Calergi, L-1359, Luxembourg. E-mail: benteng.zou@uni.lu. 


\section{Introduction}

The recent years have noticed the emergence of numerous debates on the opportunity and timing of very different types of transitions, each associated with a bunch of academic works in the economic and operational research literatures: environmental transitions (in particular the energy transition, see the early work of Tsur and Zemel, 2003), political transitions (among others, transition to democracy following Acemoglu and Robinson, 2006), organizational transitions (either in markets or in the workplace, see Vallée and Moreno, 2011) etc...In most of these works, the optimal timing of transitions (if any) are only implicitly tackled though the vast majority of the models developed are dynamic. ${ }^{1}$

There exist however an increasing number of papers interested in optimal regime transition and the inherent timing. As a common feature, all these papers use multistage optimal control techniques, first developed by Tomiyama (1984). This notably covers technological regimes switching (Boucekkine et al., 2004, or Saglam, 2011) and environmental transitions (see Boucekkine et al., 2013, or Moser et al., 2014). ${ }^{2}$ Not surpisingly, given the nature of problems tackled in the latter literature, the decisions are taken by a single player, say the central planner. However, in many cases, from one stage to another, the decision makers may differ. For example, from dictatorship to democracy, we have to move from a regime in which initially almost all the decisions are taken by a dictator, to a regime in which at the very least, the decisions are taken in a more collective way (see Acemoglu and Robinson, 2006, in a frame without multi-stage optimal control, and Boucekkine et al., 2016, with multi-stage optimal control). Similar considerations arise when analyzing international climate agreements processes where we typically switch from a regime with country-level decision making and no cooperation, for example prior to the 2015 Paris agreement, to a regime of institutional cooperation with joint decision making. On a more technical ground, the

\footnotetext{
${ }^{1}$ This is specially true in the political transitions literature, with the notable exception of Boucekkine et al., 2016.

${ }^{2}$ Applications to macroeconomic policy switching, e.g. Zampolli et al. (2016), or to workplace organization as in the above cited paper by Vallée and Moreno, 2011, can be also found.
} 
economic literature is rather very poor in papers merging multi-stage optimal control and dynamic games ingredients. The corresponding operational research literature is less poor (see for example Boucekkine et al., 2011).

In this paper, we shall deal with a generic regime switching problem where the decision making process is not the same from a regime to another. Precisely, we consider a simple model of optimal switching from competition to cooperation. We believe this problem is generic enough to cover a large set of problems. In addition to the dawn of multinational agreements, it can be also applied to political parties or companies mergers within a country. ${ }^{3}$ Needless to say, the reverse can happen, and one can notice situations in which long time cooperation comes to an end and a further competition regime sets in. Canada in 2011 pulled out of the Kyoto protocol, USA withdrew from the Paris Agreement in 2017, and it is very likely that the UK will brexit by the end of January 2020. In this kind of situation, the decision making process changes as well.

A quite rich set of questions arises from the examples given above: what are the tradeoffs involved in the decision to move from competition to cooperation (and vice versa), and what is the optimal timing for the institutional regime change if any? Since addressing these questions involves dealing with strategic tradeoffs in dynamic settings, it implies embedding dynamic games ingredients in multi-stage optimal control problems. As outlined above, the economic literature is rather thin in this respect. There is a substantial applied game theory literature of endogenous coalition formation (see for example Di Bartolomeo et al (2006), who study how coalitions among fiscal and monetary authorities are formed and what are their effects on the stabilization of output and prices). However, it generally pays no attention to the regime switching problem described above. ${ }^{4}$

\footnotetext{
${ }^{3}$ For example, in December 2003, the Progressive-Conservative Party and the Reform/Canadian Alliance parties merged and created a new right-wing political formation, the Conservative Party of Canada. In October 2007, the two most important Italian left-wing parties merged into a single political entity, the Democratic Party.

${ }^{4}$ Also, and even more clearly, our research questions and inherent settings are quite different from the classical literature on cooperative and noncooperative R\&D (D'Aspremont and Jacquemin, 1988; Suzumura, 1992), research joint ventures (Kamien, Muller and Zang, 1992) or the empirical work of Cassiman
} 
In this paper, we propose a preliminary exploration of the latter switching problem. To this end, we solve a two-stage optimal control problem. In the first stage, two players "compete" on a common state variable which could be a public good or a public bad. They engage in a dynamic game in this first stage, and we solve for openloop strategies. Arguably, under the current setting, the interesting equilibrium is the path strategies, i.e., open-loop strategies, given the fact that usually the commitment extend over the entire future time and negotiation results depend on the initial condition. Thus, the strategy may not be subgame perfect by definition, which gives possibility for changing in some future time. The decision rule strategies, i.e., the Markovian strategies, are subgame perfect Nash equilibria, but no commitment at all is possible, thus does not fit our examples above. As the state equation and the individual payoffs are linear-quadratic, the resulting dynamic game would be a conceptually trivial problem if no perspective of switching to a cooperative regime emerges. We do introduce such a possibility with the associated joint optimization of the sum of the individual payoffs.

The paper is organized as follows. Section 2 briefly presents the differential game setting. Section 3 provides the open-loop strategies during the non-cooperate and cooperative periods, thus the optimal switching conditions can be obtained. In Section 4, we turn to the numerical analysis that illustrates and deepens the theoretical findings. Section 5 provides extra discussion of the other direction of the game, that is, from cooperate to noncooperative, and Section 6 concludes.

\section{A simple model of optimal switching}

There are two players: players 1 and 2, who share a common variable, $y \in[0, Y]$, which could be public good or public bad. Each player chooses the level of a variable $x_{i} \in[0, X] \subset[0,+\infty)$, which provides her with utility. At the same time, their choice increases the level of $y$, which induces a loss in utility. Let us assume that at time 0 , players play a non-cooperative dynamic game, choosing their optimal trajectory for

and Veugelers (2002). Essentially, these models are static and can not show switching conditions from noncooperative to cooperative games and vise versa. 
$x_{i}$. Then, since their individual choices affect equally the common variable, they could decide to switch strategies at a time $T$ and continue playing strategically. Indeed, in this paper players can optimally choose a time $T$ to start playing cooperatively.

Let us provide an example from Economics that will accompany us throughout the paper. We can assume that there exists a unique final good, which requires only a polluting resource as input. With a quantity $x_{i}$ of pollution, the firm produces an amount $a_{i} x_{i}$ of the final good. Consumption provides the player with utility, but at the same time it increases the level of $\mathrm{CO}_{2}$ emissions, $y$. Obviously, the level of $\mathrm{CO}_{2}$ affects both players. In the end, the player can obtain utility directly from the consumption of $x_{i}$, but she also suffers from pollution, so she will receive disutility from $y$.

The objective of player $i=1,2$ is to maximize overall welfare, defined as

$$
\max _{x_{i}} W_{i}=\int_{0}^{+\infty} e^{-r t}\left[a_{i} x_{i}-\frac{x_{i}^{2}}{2}-\frac{b_{i} y^{2}}{2}\right] d t,
$$

which depends on her individual choice, $x_{i}$ and which is subject to the dynamic constraint:

$$
\dot{y}(t)=x_{i}+x_{j}-\delta y(t), \quad i, j=1,2 ; \quad i \neq j, \quad y(0)=y_{0} \text { given. }
$$

Welfare is the sum of instantaneous utility from time zero. Instantaneous utility at a time $t \geq 0$ is given by $a_{i} x_{i}-\frac{x_{i}^{2}}{2}-\frac{b_{i} y^{2}}{2}$. As mentioned, $x_{i}$ is the choice of player $i$. The cost of choosing $x_{i}$ is $\frac{x_{i}^{2}}{2}$, thus the net gain from $x_{i}$ is $a_{i} x_{i}-\frac{x_{i}^{2}}{2}$, with $a_{i}(\geq X)$ positive constant measuring the unit gain.

An increase in the state variable $y$ has a negative effect on utility. Indeed, as in our example where $y$ is the stock of $\mathrm{CO}_{2}$ emissions, then any increase in $y$ induces a damage $\frac{b_{i} y^{2}}{2}$, with scaling parameter $b_{i}$, a positive constant.

Suppose that at some future date $T$, the two players decide to play cooperatively. Then, the join objective is

$$
\max _{x_{i}, x_{j}} W_{I I}(T)=\int_{T}^{+\infty} e^{-r t}\left[a_{i} x_{i}+a_{j} x_{j}-\frac{x_{i}^{2}+x_{j}^{2}}{2}-\frac{\left(b_{i}+b_{j}\right) y^{2}}{2}\right] d t,
$$

subject to the same state equation (1).

For simplicity reasons, we only consider here the symmetric case where $a_{i}=a_{j}=a$ and $b_{i}=b_{j}=b$. 
Remark 1 The difficulty of considering the asymmetric case is to provide the sharing principal in the second period. Here, in the symmetric case, the two share equally the gain.

In the following, we compute the optimal switching time $T$. Denote by $W_{I}(T)$ welfare of a player playing non-cooperative, from time 0 to the switching time $T$, when she starts playing cooperatively. Then, the player solves the following problem

$$
\max _{x_{i}} W_{I}(T)=\int_{0}^{T} e^{-r t}\left[a_{i} x_{i}-\frac{x_{i}^{2}}{2}-\frac{b_{i} y^{2}}{2}\right] d t,
$$

subject to the law of motion (1).

The optimal choice of $T$ is given by the solution of

$$
\max _{T}\left[W_{I}(T)+e^{-r T} \frac{W_{I I}(T)}{2}\right] .
$$

If $T=0$, then it is optimal for the two players to play cooperatively immediately; while if $T=+\infty$, the noncooperative game should continue forever.

\section{The optimal switching strategy}

As in Boucekkine et al. (2004) and the references after them, we solve first the second stage problem. Optimal trajectories for $x$ and $y$ are obtained in case the players play cooperatively from $T$ onwards. Second, taking $T$ as given, we solve the first noncooperative game. We obtain optimal trajectories in both phases that depend on $y_{0}$ and $T$. Finally, the optimal switching time $T$ is obtained substituting the resulting $W_{I}$ and $W_{I I}$ into (2), and solving the resulting problem. As a result, the optimal solution will be made of an optimal switching time $T$, and the optimal trajectories for $y$ and $x$ before and after the switch.

\subsection{The cooperative regime}

The joint symmetric optimization problem can be restated as:

$$
\max _{x} W_{I I}(T)=\int_{T}^{+\infty} e^{-r t}\left[2 a x-x^{2}-b y^{2}\right] d t,
$$


subject to the state equation

$$
\dot{y}(t)=2 x-\delta y(t)
$$

for $i, j=1,2, i \neq j$, and where $y(0)=y_{0}$ is given. Define the associated Hamiltonian as

$$
\mathcal{H}_{I I}\left(x, y, \lambda_{I I}\right)=2 a x-x^{2}-b y^{2}+\lambda_{I I}(2 x-\delta y(t))
$$

with $\lambda_{I I}$ the co-state variable. The first order conditions yield the following set of optimal conditions

$$
\left\{\begin{array}{l}
x_{I I}=a+\lambda_{I I}, \\
\dot{y}_{I I}(t)=-\delta y+2 \lambda_{I I}+2 a, \\
\dot{\lambda}_{I I}(t)=2 b y+(r+\delta) \lambda_{I I},
\end{array}\right.
$$

with transversality condition $\lim _{t \rightarrow+\infty} e^{-r t} y(t) \lambda_{I I}(t)=0$.

Next proposition provides the analytical solution to (4). We do not reproduce here the proof since it is a standard exercise.

Proposition 1 Suppose that the two players play the cooperative game from time T onwards, then the optimal effort is given by (4). The corresponding state and costate variables are given by:

$$
\left\{\begin{array}{l}
y_{I I}(t)=2 C_{1} e^{\mu t}+\bar{y} \\
\lambda_{I I}(t)=(\mu+\delta) C_{1} e^{\mu t}+\bar{\lambda}
\end{array}\right.
$$

where $t \geq T$ and $\mu=\frac{r-\sqrt{r^{2}+4[\delta(r+\delta)+4 b]}}{2}(<0)$. Constant $C_{1}$ will be determined later by the transversality condition $y\left(T^{-}\right)=y\left(T^{+}\right)$.

Moreover, there exists a unique steady state $(\bar{y}, \bar{\lambda})$ given by

$$
\bar{y}=\frac{2 a(r+\delta)}{\delta(r+\delta)+4 b}, \quad \bar{\lambda}=-\frac{4 a b}{\delta(r+\delta)+4 b} .
$$

Associated to this steady state, we can compute the steady state of $x_{I I}$,

$$
\bar{x}=a+\bar{\lambda}=a\left(1-\frac{4 b}{\delta(r+\delta)+4 b}\right) .
$$

Corollary 1 Both $\bar{y}$ and $\bar{x}$ increase with a, they decrease with $b$. 
Obviously if $T=0$, that is, if the cooperation starts from the beginning, then $y(T)=$ $y(0)=y_{0}$ and $C_{1}=\frac{y_{0}-\bar{y}}{2}$. It is easy to check that the optimal joint welfare is in this case

$$
W_{I I}^{*}(0)=\frac{2 a(a+\bar{\lambda})-(a+\bar{\lambda})^{2}-b \bar{y}}{r}+\frac{(\mu+\delta)^{2} C_{1}^{2}}{2 \mu-r}+\frac{2 C_{1}(b+(\mu+\delta) \bar{\lambda})}{\mu-r} .
$$

\subsection{The non-cooperative regime}

Let us solve the first period non-cooperative game for a switching time $T$ given. The first period optimization problem for player $i$ is

$$
\max _{x_{i}} W_{I}(T)=\int_{0}^{T} e^{-r t}\left[a_{i} x_{i}-\frac{x_{i}^{2}}{2}-\frac{b_{i} y^{2}}{2}\right] d t,
$$

subject to

$$
\dot{y}(t)=x_{i}+x_{j}-\delta y(t),
$$

for $i, j=1,2, i \neq j$, and where $y(0)=y_{0}$ is given. The associated Hamiltonian is

$$
\mathcal{H}_{I}\left(x_{i}, y, \lambda_{I}\right)=a_{i} x_{i}-\frac{x_{i}^{2}}{2}-\frac{b_{i} y^{2}}{2}+\lambda_{I}\left(x_{i}+x_{j}-\delta y(t)\right),
$$

with $\lambda_{I}$ co-state variable in the first period. In the symmetric setting, the first order conditions yield the following set of optimal conditions:

$$
\left\{\begin{array}{l}
x_{I}=a+\lambda_{I}, \\
\dot{y}_{I}(t)=-\delta y_{I}+2 \lambda_{I}+2 a, \\
\dot{\lambda}_{I}(t)=b y+(r+\delta) \lambda_{I},
\end{array}\right.
$$

Proposition 2 below provides the analytical solution to the first order conditions in (8). Its proof can be found in the appendix.

Proposition 2 Suppose that at time $T$, the two players start playing the cooperative game. Then, for $t \in[0, T]$, the unique symmetric strategic Nash equilibrium is $x_{I}(t)=a+\lambda_{I}$, with state, $y_{I}$, and costate variables, $\lambda_{I}$, given by

$$
\left\{\begin{array}{l}
y_{I}(t)=\hat{a} e^{\nu_{1} t}+\hat{b} e^{\nu_{2} t}+y_{P}(t), \\
\lambda_{I}(t)=\hat{c} e^{\nu_{1} t}+\hat{d} e^{\nu_{2} t}+\lambda_{P}(t),
\end{array}\right.
$$


in which $\nu_{1}$ and $\nu_{2}$ are eigenvalues given by

$$
\nu_{1}=\frac{r-\sqrt{r^{2}+4[\delta(r+\delta)+2 b]}}{2}, \quad \nu_{2}=\frac{r+\sqrt{r^{2}+4[\delta(r+\delta)+2 b]}}{2} .
$$

$\left(y_{P}(t), \lambda_{P}(t)\right)$ is a special solution:

$$
\begin{aligned}
& y_{P}(t)=\hat{y}_{P}\left(1-e^{\nu_{1} t}\right)+\bar{y}_{P}\left(1-e^{\nu_{2} t}\right), \\
& \lambda_{P}(t)=\hat{\lambda}_{P}\left(1-e^{\nu_{1} t}\right)+\bar{\lambda}_{P}\left(1-e^{\nu_{2} t}\right),
\end{aligned}
$$

with

$$
\begin{aligned}
& \hat{y}_{P}=\frac{2 a\left(\nu_{2}+\delta\right)}{\nu_{1}\left(\nu_{1}-\nu_{2}\right)}, \quad \bar{y}_{P}=-\frac{2 a\left(\nu_{1}+\delta\right)}{\nu_{2}\left(\nu_{1}-\nu_{2}\right)}, \\
& \hat{\lambda}_{P}=\frac{a\left(\nu_{1}+\delta\right)\left(\nu_{2}+\delta\right)}{\nu_{1}\left(\nu_{1}-\nu_{2}\right)}, \quad \bar{\lambda}_{P}=-\frac{a\left(\nu_{1}+\delta\right)\left(\nu_{2}+\delta\right)}{\nu_{2}\left(\nu_{1}-\nu_{2}\right)} .
\end{aligned}
$$

Constants $\widehat{a}, \widehat{b}, \widehat{c}, \widehat{d}$ are given by

$$
\widehat{a}=\frac{-\left(\nu_{2}+\delta\right) y_{0}+2 \lambda_{0}}{\nu_{1}-\nu_{2}}, \widehat{b}=\frac{\left(\nu_{1}+\delta\right) y_{0}-2 \lambda_{0}}{\nu_{1}-\nu_{2}}
$$

and

$$
\widehat{c}=\frac{\left(\nu_{1}+\delta\right)\left[-\left(\nu_{2}+\delta\right) y_{0}+2 \lambda_{0}\right]}{2\left(\nu_{1}-\nu_{2}\right)}, \widehat{d}=\frac{\left(\nu_{2}+\delta\right)\left[\left(\nu_{1}+\delta\right) y_{0}-2 \lambda_{0}\right]}{2\left(\nu_{1}-\nu_{2}\right)},
$$

where $y_{0}$ is given initial condition, while $\lambda_{0}$ will be determined by the switching condition at time $T$.

A special case arises when $T=+\infty$, that is, when no cooperation is possible. Here the solution of (8) with transversality condition $\lim _{t \rightarrow+\infty} e^{-r t} y(t) \lambda_{I}(t)=0$, is

$$
\left\{\begin{array}{l}
y_{I}(t)=2 C_{1 I} e^{\nu_{1} t}+\bar{y}_{I}, \\
\lambda_{I}(t)=C_{1 I} e^{\nu_{1} t}+\bar{\lambda}_{I}
\end{array}\right.
$$

where $C_{1 I}=\frac{y_{0}-\bar{y}_{I}}{2}$ and the steady state $\left(\bar{y}_{I}, \bar{\lambda}_{I}\right)=\left(\frac{2 a(r+\delta)}{\delta(r+\delta)+2 b},-\frac{2 a b}{\delta(r+\delta)+2 b}\right)$. In this special case, each player's social welfare is

$$
W_{I}^{*}(+\infty)=\frac{a\left(a+\bar{\lambda}_{I}\right)-\left(a+\bar{\lambda}_{I}\right)^{2} / 2-b \bar{y}_{I} / 2}{r}+\frac{\left(\nu_{1}+\delta\right)^{2} C_{1 I}^{2}}{2\left(2 \nu_{1}-r\right)}+\frac{C_{1 I}\left(b+\left(\nu_{1}+\delta\right) \bar{\lambda}_{I}\right)}{\nu_{1}-r} .
$$


From (6) and (11), it is easy to check that

$$
\frac{W_{I I}^{*}}{2}(+\infty)-W_{I}^{*}(+\infty) \lesseqgtr 0,
$$

depending on the combination of parameters. In other words, it is possible that the switching happens at $T=0$, or $T=+\infty$, or $T \in(0,+\infty)$, depending on the situation under study, which can also be seen in the Section 4 with numerical illustration.

\subsection{Optimal switching time}

At the switching time, $T$, and by continuity, the state variable $y$ must verify that

$$
y_{I}(T)=y_{I I}(T) .
$$

Similarly, the costate variable must verify that

$$
\lambda_{I}(T)=\lambda_{I I}(T) .
$$

Usually, as in Boucekkine et al $(2004,2013,2016)$, in the study of the optimal switching in optimal control problems with a unique decision maker, a transversality condition is imposed on the maximized Hamiltonian at the switching time $T$. In that case, the maximized Hamiltonian is exactly the same immediately before and immediately after the switch.

However, under the current dynamic game setting, it is different. Before and after the switch, there are different decision makers as stated in the Introduction. Before the switch, each individual player takes her own optimal decisions, while after the switch, it is a join choice. Thus, it is improper to equalize the maximized Hamiltonian before and after the switch, even with identical players.

Therefore, we impose that the shadow values are the same before and after the switching. In our example where $y$ measures the stock of $\mathrm{CO}_{2}$, this transversality condition implies that the shadow value of pollution does not change immediately due to the signature of some agreement or protocol. Instead it takes some time for the shadow value to change, while immediate changes come from the choice variables. 
Combining (12) and (13), we obtain that the values of $\lambda_{0}(T)$ and $C_{1}(T)$ :

$$
\left\{\begin{array}{l}
\lambda_{0}(T)=\frac{2 E_{1}(T)-(\mu+\delta) A_{1}(T)}{(\mu+\delta) A_{2}(T)-2 E_{2}(T)}, \\
C_{1}(T)=\frac{E_{1}(T)+E_{2}(T) \lambda_{0}}{\mu+\delta} e^{-\mu T},
\end{array}\right.
$$

where

$$
A_{1}(t)=\frac{\left(\nu_{1}+\delta\right) e^{\nu_{2} t}-\left(\nu_{2}+\delta\right) e^{\nu_{1} t}}{\nu_{1}-\nu_{2}} y_{0}+\left(y_{P}(t)-\bar{y}\right), \quad A_{2}(t)=\frac{2\left(e^{\nu_{1} t}-e^{\nu_{2} t}\right)}{\nu_{1}-\nu_{2}},
$$

and

$E_{1}(t)=\frac{\left(\nu_{1}+\delta\right)\left(\nu_{2}+\delta\right)\left(e^{\nu_{2} t}-e^{\nu_{1} t}\right)}{2\left(\nu_{1}-\nu_{2}\right)} y_{0}+\left(\lambda_{P}(t)-\bar{\lambda}\right), \quad E_{2}(t)=\frac{\left(\nu_{1}+\delta\right) e^{\nu_{1} t}-\left(\nu_{2}+\delta\right) e^{\nu_{2} t}}{\nu_{1}-\nu_{2}}$.

Thus, substituting (14) into (9) and (5), we could obtain the explicit optimal trajectories in both periods.

The optimal switching time $T$ is given by the optimization problem (2). With the above explicit solutions, it only remains to calculate the social welfare in the two periods, i.e. $W_{I}(T)$ and $W_{I I}(T)$. It is easy to check that

$$
\begin{aligned}
\frac{e^{-r T}}{2} W_{I I}= & \left(\frac{a^{2}-\bar{\lambda}^{2}-b \bar{y}^{2}}{2}\right) \frac{e^{-2 r T}}{r}-[(\mu+\delta) \bar{\lambda}+2 b \bar{y}] C_{1} \frac{e^{(\mu-2 r) T}}{r-\mu} \\
& -\left[\frac{(\mu+\delta)^{2}+4 b}{2}\right] C_{1}^{2} \frac{e^{(2 \mu-2 r) T}}{r-2 \mu},
\end{aligned}
$$

in which $C_{1}=C_{1}(T)$ is given by (14). 
Similarly, the first period social welfare $W_{I}$ is given by

$$
\begin{aligned}
W_{I}= & {\left[\frac{a^{2}-\left(\widehat{\lambda}_{P}+\bar{\lambda}_{P}\right)^{2}-b\left(\widehat{y}_{P}+\bar{y}_{P}\right)^{2}}{2}\right] \frac{\left(e^{-r T}-1\right)}{-r} } \\
& -\left[\frac{\left(\widehat{c}-\widehat{\lambda}_{P}\right)^{2}}{2}+\frac{b\left(\widehat{a}-\widehat{y}_{P}\right)^{2}}{2}\right] \frac{\left(e^{\left(2 \nu_{1}-r\right) T}-1\right)}{2 \nu_{1}-r} \\
& -\left[\frac{\left(\widehat{d}-\bar{\lambda}_{P}\right)^{2}}{2}+\frac{b\left(\widehat{b}-\bar{y}_{P}\right)^{2}}{2}\right] \frac{\left(e^{\left(2 \nu_{2}-r\right) T}-1\right)}{2 \nu_{2}-r} \\
& -\left[\left(\widehat{c}-\widehat{\lambda}_{P}\right)\left(\widehat{d}-\bar{\lambda}_{P}\right)+b\left(\widehat{a}-\widehat{y}_{P}\right)\left(\widehat{b}-\bar{y}_{P}\right)\right] \frac{\left(e^{\left(\nu_{1}+\nu_{2}-r\right) T}-1\right)}{\nu_{1}+\nu_{2}-r} \\
& -\left[\left(\widehat{c}-\widehat{\lambda}_{P}\right)\left(\widehat{\lambda}_{P}+\bar{\lambda}_{P}\right)+b\left(\widehat{a}-\widehat{y}_{P}\right)\left(\widehat{y}_{P}+\bar{y}_{P}\right)\right] \frac{\left(e^{\left(\nu_{1}-r\right) T}-1\right)}{\nu_{1}-r} \\
& -\left[\left(\widehat{d}-\bar{\lambda}_{P}\right)\left(\widehat{\lambda}_{P}+\bar{\lambda}_{P}\right)+b\left(\widehat{b}-\bar{y}_{P}\right)\left(\widehat{y}_{P}+\bar{y}_{P}\right)\right] \frac{\left(e^{\left(\nu_{2}-r\right) T}-1\right)}{\nu_{2}-r},
\end{aligned}
$$

in which $\widehat{a}, \widehat{b}, \widehat{c}, \widehat{d}$ depend on the switching time $T$ as well, via $\lambda_{0}(T)$.

The first order condition from (2) yields

$$
\frac{d W_{I}(T)}{d T}+\frac{e^{-r T}}{2}\left[-r W_{I I}(T)+\frac{d W_{I I}(T)}{d T}\right]=0 .
$$

Intuitively, the first term should be nonnegative, i.e. the longer the time period $[0, T]$, the higher the welfare it yields (otherwise $T=0$ already). The second term should be non-positive (otherwise $T=+\infty$ ). Nevertheless, due to the complexity of expressions (15) and (16), the study of an explicit form becomes cumbersome, and the study of the properties and impacts become impossible. Thus, it is not wise to continue working on the search of explicit solutions. Instead, we focus in next section on numerical simulations to illustrate the impacts of important parameters, such as, the efficient parameter, $a$, and dis-utility parameter, $b$, as well as the initial condition, $y_{0}$. Although the model is not realistically calibrated, the qualitative pattern is illustrative.

\section{Numerical illustration}

This section illustrates numerically the theoretical results obtained in the previous sections. First, we compare the optimal switching time as a function of the initial endow- 
ment of the state variable, $y_{0}$. In a second set of exercises, we compute the optimal dynamic trajectories of the state variable $y$ and of the optimal choice $x$.

\subsection{Optimal switching time}

To seize the importance of all the elements of the model, we have computed the optimal switching time under various scenarios for both $a$ and $b$. In all exercises, $r=0.0015$ and $\delta=0.01$. The time discount is in line with the most recent literature (see the literature following Stern, 2006).

We begin by exploring the role of $a$. Setting $b=0.000095$ we choose an economy which loses little utility with $y$. Two economies are compared: a performant economy, which can extract high utility from the same amount of the choice variable $x$, for which $a=0.115$; and a second less efficient economy, in which $a=0.109$. Figure 1 shows the optimal switching time and the associated overall welfare when the initial endowment of the state variable ranges from $y_{0}=50$ to $y_{0}=100 .^{5}$
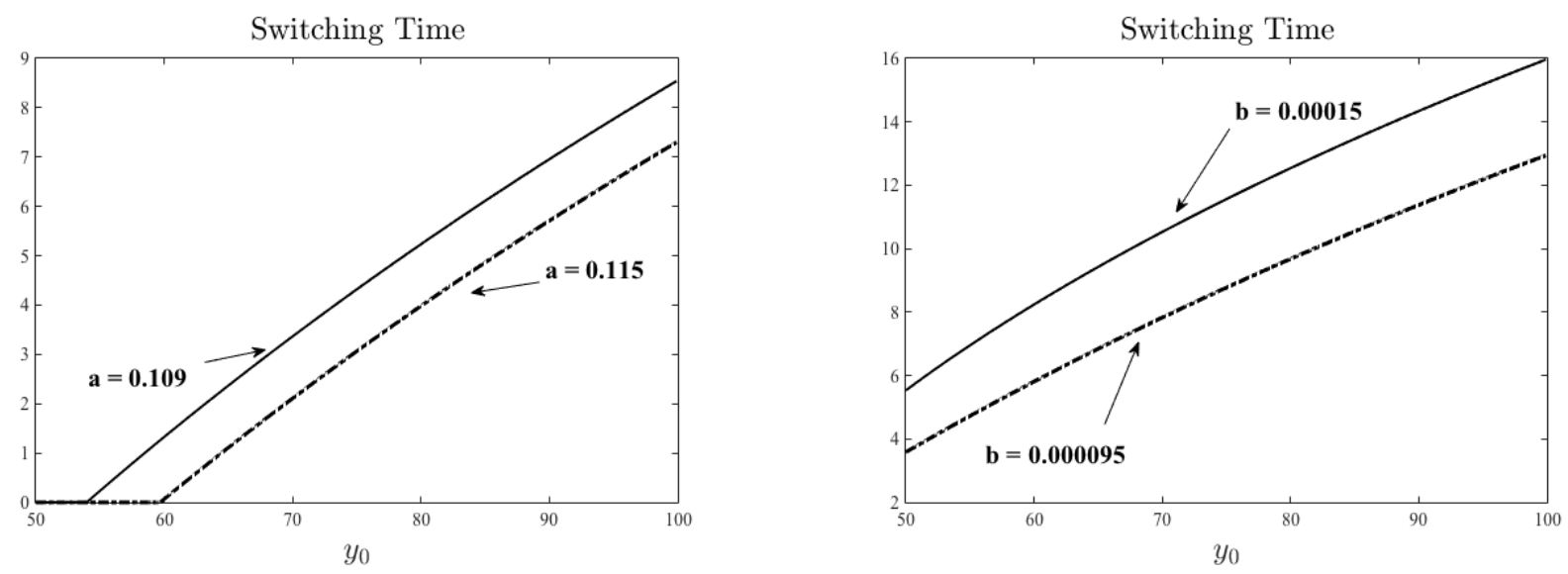

Figure 1: Optimal T. Left: role of $a$ when $b=0.000095$. Right: role of $b$ when $a=0.09$.

Interpreting $y$ as the $\mathrm{CO}_{2}$ stock and $x$ as consumption, $a$ is a measure of the effi-

\footnotetext{
${ }^{5}$ The switching time trajectories displayed in Figures 1 and 2 are maxima of (2), since they verify the second order condition as shown in Appendix B.
} 
ciency of the economy. Indeed, with the same amount of the resource, the higher $a$, the more of the final consumption good we obtain. Low levels of $y_{0}$ correspond to economies that are not too polluted initially. By the same token, high $y_{0}$ correspond to economies that are already very polluted at the beginning of the game.

On the left panel of Figure 1, we see that players always decide to play cooperatively, that is $0 \leq T<\infty$. They cooperate from $T=0$ when $y_{0}$ is relatively low. When the environmental quality is high, players join as soon as possible to preserve the environment and avoid the loss of utility induced by pollution. Besides, switching times are increasing functions of the initial stock of $y_{0}$. If $y$ is the stock of $\mathrm{CO}_{2}$ emissions, then clean economies start cooperating earlier. Indeed, earlier cooperation reduces global emissions and ensures a higher production in the future. Conversely, when economies are relatively dirtier, $y_{0}$ is relatively high, then they start cooperating later as if the damage was already too large to struggle against. Finally, note that $T$ is always smaller for $a=0.115$. Efficient economies cooperate sooner since their advantage in production allows them to obtain more of the final good producing the same amount of pollution. Note that better performing economies can cope better with possible losses in consumption arising upon cooperation.

Let us study next the negative effect of the state variable in utility, as captured by parameter $b$. The right panel in Figure 1 shows optimal switching times when $a=0.09$ for two values of $b$ : a high level of $b=0.00015$ and a low level $b=0.000095$. Again, understanding $y$ as $\mathrm{CO}_{2}$ emissions, an economy with a low $b$ corresponds to an economy less sensitive to pollution, such as a very small open economy or an economy embodied with sufficient forest or water source to absorb important amounts of $\mathrm{CO}_{2}$. These economies start playing cooperatively earlier in order to maintain $y$ at a moderate level.

\subsection{Optimal dynamic trajectories}

Next, let us illustrate the dynamic trajectories for the economy underlining the roles of $a$ and $b$ in the dynamics of the optimal control and state variables. In the first exercise, $a=0.5$ and we compare two economies which differ in their sensitivity to pollution. 
In the less sensitive $b=0.000095$ and in the more sensitive $b=0.00015$. In both cases the economy is initially endowed with $y_{0}=75$. Figure 2 shows our results for the state variable, $y$, and for the control variable per capita, $x$.
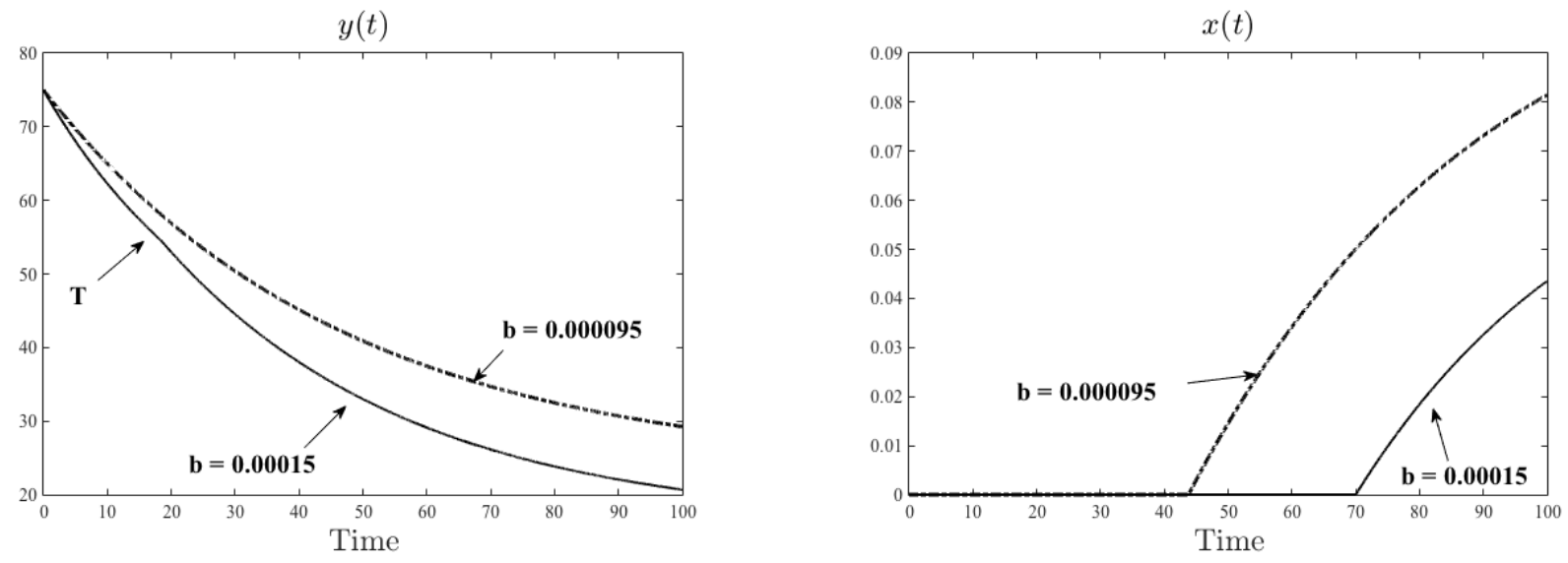

Figure 2: Dynamics of $y(\cdot)$ and $x(\cdot) . a=0.5$.

The results are in line with the precedent theoretical analysis. Indeed, we knew that the steady state of $y, y_{s s}$, decreases with $b$. Hence the long-term of $y$ for $b=0.000095$ is higher than for $b=0.00015$. The less sensitive economy can maintain a higher production level and afford higher consumption because it loses less welfare from $\mathrm{CO}_{2}$ emissions. When $b$ is low, players start playing cooperatively from the beginning and the economy accumulates more pollution.

The right panel of Figure 2 reveals the thinking of the policy maker. Compared to their steady states, both economies start with a highly polluted environment, which makes them lose a significant amount of welfare. Hence, the policy maker decides not to consume during a certain period of time. This is true when $b=0.000095$ and that players play together from $t=0$, as well as in the more sensitive case, when $b=0.00015$, and that there are two policy makers from $t=0$ till $T=18.4$. Note that the less sensitive economy starts consuming before and it consumes more.

Our last exercise compares two economies which differ in their efficiency level letting $b=0.000095$. In the first, $a=0.5$, and in the second, the efficiency level doubles 
the first, $a=1$. As shown in Proposition 1, the steady state of $y$ keeps the same proportion than efficiency. Indeed, $y_{s s}=23.23$ when $a=0.5$ and $y_{s s}=46.46$ when $a=1$. Our results are displayed in Figure 4. The most salient feature is that the most efficient economy does not sacrifice initial consumption in order to preserve $y$ at a low level. Its technological advantage allows the policy maker to choose positive levels of consumption from $t=0$.
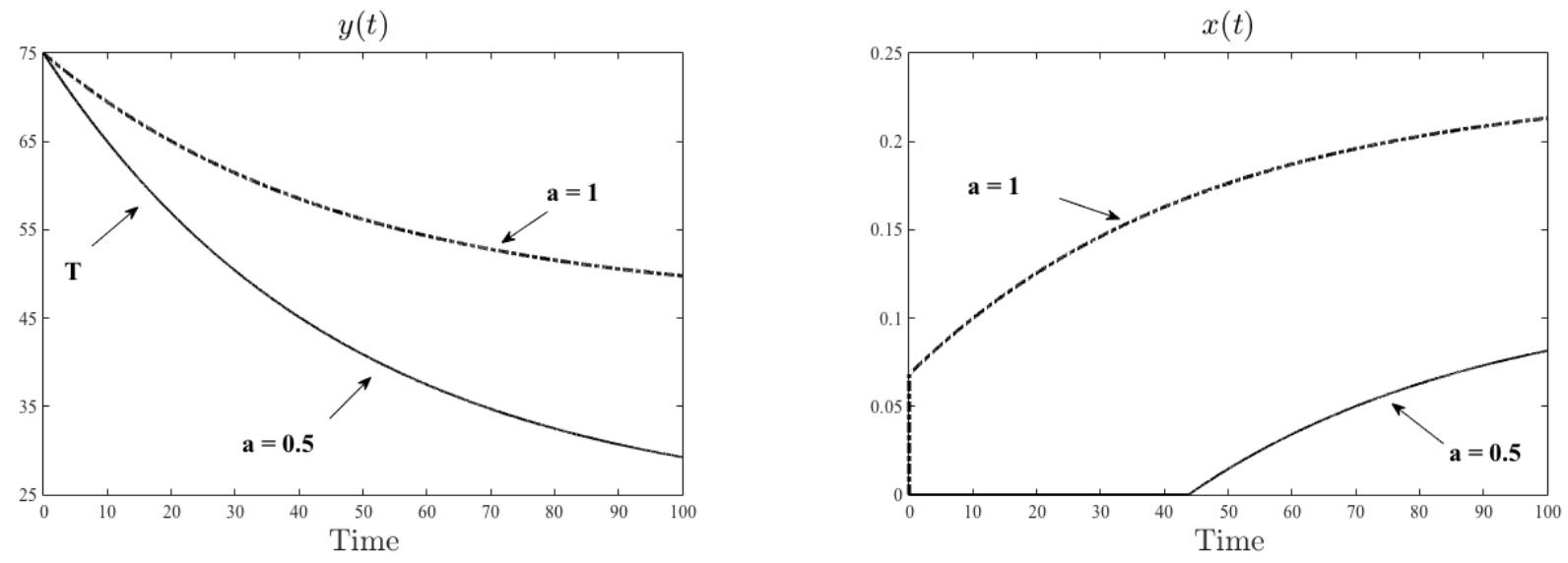

Figure 3: Dynamics of $y(\cdot)$ and $x(\cdot) . b=0.000095$.

\section{From the cooperative to the non-cooperative game}

In the previous sections, if players decide to join at time $T$, they will play cooperatively forever after. In other words, their commitment to some protocol, or union, or agreement is irreversible. However, if it was possible to exit in some future time, choices and trajectories would be more complicated. In this section, we extend the previous study to include the possibility of a future exit. The steps of the game are the following:

Step 1. The two players play the non-cooperative differential game until time $T_{1}$. Under the symmetric assumption, the revenue of the identical players is $V_{I}\left(T_{1}, T_{2}\right)$

Step 2. From time $T_{1}$ until $T_{2}$, the two players play cooperatively. Denote the join revenue as $V_{I I}\left(T_{1}, T_{2}\right)$. 
Step 3. One of the two players exits the cooperative game at $T_{2}$, thus from $T_{2}$ onwards, the two players play the non-cooperative differential games again. Denote the revenue in this period as $V_{I I I}\left(T_{1}, T_{2}\right)$.

Then the optimal choice of switching times should be given by

$$
\max _{T_{1}, T_{2}}\left[V_{I}\left(T_{1}, T_{2}\right)+e^{-r T_{1}} V_{I I}\left(T_{1}, T_{2}\right)+e^{-r T_{2}} V_{I I I}\left(T_{1}, T_{2}\right)\right] .
$$

Mathematically, this more complicated differential game can be solved by backward induction by reversing the calculation order of the above Section 3. Similarly, numerical investigation can be performed the same way as Section 4 . Therefore, we do not need to do this exercise here in detail.

\section{Conclusion}

We investigate both theoretically and numerically some dynamic game settings where players choose to switch from noncooperative competition to cooperate (or vise versa) and which automatically change the decision makers as well. The explicit conditions of open-loop strategic switching conditions are presented, though it can not be fully analytical. We rely on numerical simulation and demonstrate the importance of parameters. Not surprising, given the intrinsic nature of open-loop strategies, both switching time and the social welfare depend essentially on the initial condition of the game, but neither were monotonic. The efficient parameter plays very important role in not only the decision of switching from noncooperative to cooperative, but also the choice of consumption. High efficient parameter may cover the side effects, such as leading to high pollution as a by product. Of course, different damaging parameter may be introduced between non-cooperative and cooperative games, which is a future work. Furthermore, it is easy to imagine the situation where the two players are asymmetric or there are more than two players. We do believe that the current study paves the way to handle a much wider class of problems, beyond the examples we presented in the Introduction. 


\section{A Appendix. Proof of Proposition 2}

Recall the dynamic system as

$$
\left\{\begin{array}{l}
\dot{y}_{I}(t)=-\delta y_{I}+2 \lambda_{I}+2 a \\
\dot{\lambda}_{I}(t)=b y+(r+\delta) \lambda_{I}
\end{array}\right.
$$

It is easy to obtain the associated eigenvalues

$$
\nu_{1,2}=\frac{r \pm \sqrt{r^{2}+4(\delta(r+\delta)+2 b)}}{2}
$$

with $\nu_{1}<0$ and $\nu_{2}>0$, and associated eigenvectors

$$
\overrightarrow{v_{i}}=\left(\begin{array}{c}
2 \\
\nu_{i}+\delta
\end{array}\right), \quad i=1,2
$$

Define matrix

$$
M(t)=\left(\begin{array}{ll}
\overrightarrow{v_{1}} & \overrightarrow{v_{2}}
\end{array}\right)\left(\begin{array}{cc}
e^{\nu_{1} t} & 0 \\
0 & e^{\nu_{2} t}
\end{array}\right)=\left(\begin{array}{cc}
2 e^{\nu_{1} t} & 2 e^{\nu_{2} t} \\
\left(\nu_{1}+\delta\right) e^{\nu_{1} t} & \left(\nu_{2}+\delta\right) e^{\nu_{2} t}
\end{array}\right)
$$

Thus the inverse matrix of $M(t)$ is

$$
M^{-1}(t)=\left(\begin{array}{cc}
-\frac{\nu_{2}+\delta}{2\left(\nu_{1}-2\right)} e^{-\nu_{1} t} & \frac{e^{-\nu_{1} t}}{\nu_{1}-\nu_{2}} \\
\frac{\nu_{1}+\delta}{2\left(\nu_{1}-\nu_{2}\right)} e^{-\nu_{2} t} & -\frac{e^{-\nu_{2} t}}{\nu_{1}-\nu_{2}}
\end{array}\right) .
$$

The unique solution of the system is then given by

$$
\left(\begin{array}{l}
y(t) \\
\lambda(t)
\end{array}\right)=M(t) M^{-1}(0)\left(\begin{array}{c}
y_{0} \\
\lambda(0)
\end{array}\right)+M(t) \int_{0}^{t} M^{-1}(s)\left(\begin{array}{c}
2 a \\
0
\end{array}\right) d s
$$

in which $\lambda(0)$ is undetermined. Substituting $M(t), M^{-1}(t)$ and $M^{-1}(0)$ into the above matrix algebra and taking integrals, we obtain the explicit solution in Proposition 2.

That completes the proof. 


\section{B Appendix. Second order condition.}

Figure 4 shows the value of the second order derivative of (2) associated to the examples in Section 4. The graphs show that the second derivative is negative for all values of $y_{0}$ in the four examples. Hence, the switching times $T$ in Figure 1 is always a maximum.
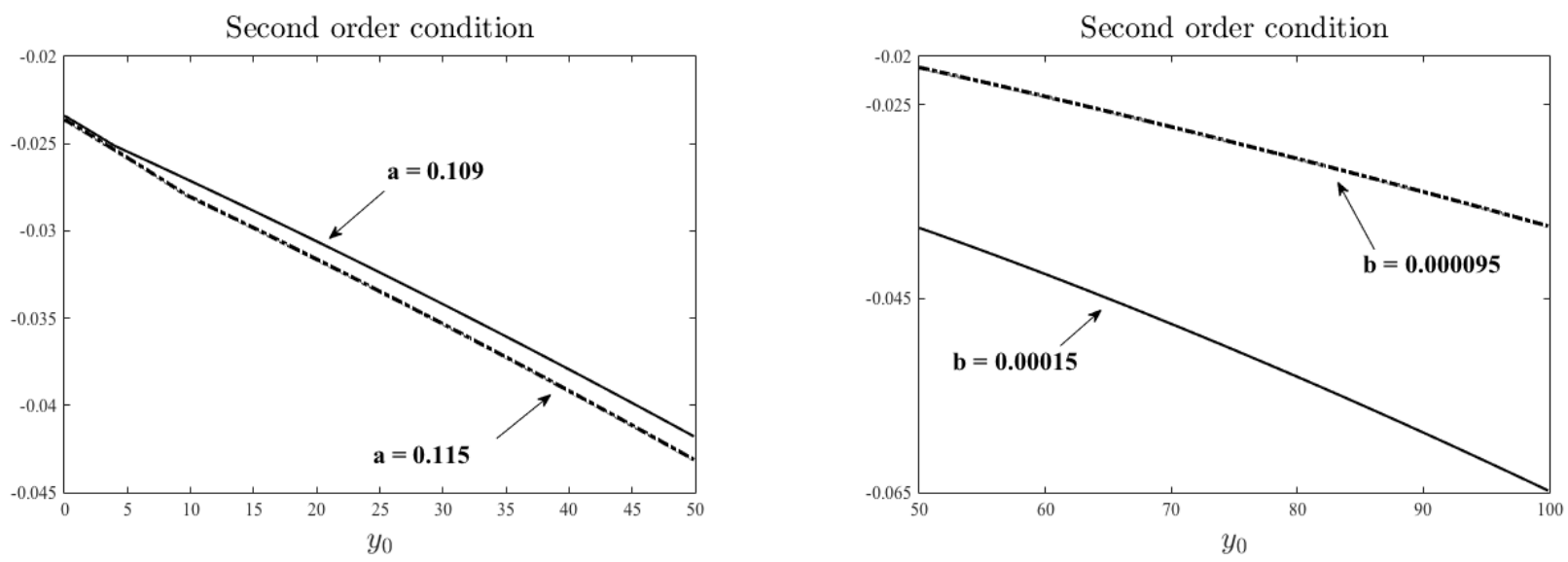

Figure 4: Second order conditions.

\section{References}

[1] Acemoglu D. and J. Robinson (2006). Economic Origins of Dictatorship and Democracy. Cambrige University Press.

[2] Boucekkine R., C. Saglam and T. Vallée (2004). Technology adoption under embodiment: A two-stage optimal control approach. Macroeconomic Dynamics 8(2), 250-271.

[3] Boucekkine R., J. Krawczyk and T. Vallée (2011). Environmental quality versus economic performance: a dynamic game approach. Optimal Control Applications and Methods 32, 29-46. 
[4] Boucekkine R., Pommeret A. and F. Prieur (2013). Optimal regime switching and threshold effects. Journal of Economic Dynamics and Control, 37, 2979-2997.

[5] Boucekkine, R., F. Prieur and K. Puzon (2016). On the timing of political regime changes in resource-dependent economies. European Economic Review 85, 188-207.

[6] Cassiman B. and R. Veugelers (2002). RED cooperation and cpillovers: Some empirical evidence from Belgium. The American Economic Review 92, 1169-1184

[7] D'Aspremont C. and A. Jacquemin (1988). Cooperative and Noncooperative R $\mathcal{E}$ D in Duopoly with Spillovers. The American Economic Review 78, 1133-1137.

[8] Di Bartolomeo G., J. Engwerda, J. Plasmans and B. van Aarle (2006). Staying together or breaking apart: policy-makers' endogenous coalitions formation in the European Economic and Monetary Union. Computers \& Operations Research 33, 438-463.

[9] Dockner E., S. Jorgensen, N. Van Long, and G. Sorger (2000). Differential Games in Economics and Management. Cambridge University Press.

[10] Kamien M. , E. Muller and I. Zang (1992). Research joint ventures and RED cartels. The American Economic Review 82, 1293-1306.

[11] Mair P (1990). The electoral payoffs of fission and fusion. British Journal of Political Science 20, 131- 42.

[12] Moser E., A. Seidel and G. Feichtinger (2014). History-dependence in productionpollution-trade-off models: a multi-stage approach. Annals of Operations Research $222,455-481$

[13] Saglam C. (2011). Optimal pattern of technology adoptions under embodiment: A multistage optimal control approach. Optimal Control Applications and Methods 32, 574586

[14] Stern N. (2006). The Economics of Climate Change: The Stern Review. Cambridge University Press. 
[15] Suzumura K. (1992). Cooperative and noncooperative RED in an oligopoly with spillover. The American Economic Review 82, 1307-1320.

[16] Tomiyama K. (1985). Two-stage optimal control problems and optimality conditions. Journal of Economic Dynamics and Control 9, 317-337

[17] Tsur Y. and A. Zemel (2003). Optimal transition to backstop substitutes for nonrenewable resources. Journal of Economic Dynamics and Control 27, 551-572

[18] Vallée T. and E. Moreno Galbis (2011). Optimal time switching from tayloristic to holistic workplace organization. Structural Change and Economic Dynamics 22, 238246

[19] Zampolli F. (2006). Optimal monetary policy in a regime switching economy: the response to abrupt shifts in exchange rate dynamics. Journal of Economic Dynamics and Control 30, 15271567. 